\title{
On Electromagnetic Radiation From a Magnetic Dipole With Arbitrary Orientation Embedded in a Lossless Magneto-Ionic Medium
}

\author{
H. Motz \\ Department of Engineering Science, University of Oxford, Oxford, England
}

(Received October 22, 1964; revised December 7, 1964)

The methods of an earlier paper for calculating the radiation from an electric dipole are extended to the case of a magnetic dipole, and numerical data concerning the radiation admittance of such a dipole embedded in an infinite magneto-ionic medium are given.

\section{Introduction}

In an earlier paper [Kogelnik 1960a] the radiation resistance matrix of an electric dipole with arbitrary orientation with respect to the magnetic field was calculated in terms of the tensor elements characterizing the medium in which it is embedded. Numerical data concerning the resistance matrix were published in the Proceedings of the Upsala Conference [Kogelnik 1960b]. The extension of the method to the case of a magnetic dipole [Kogelnik, Oxford Thesis, 1960, unpublished] was given at the Copenhagen Symposium on Antennas [Kogelnik and Motz 1963]. The purpose of this paper is to present numerical data which were not given in that paper, and to briefly but completely reformulate the theory.

\section{Definitions and Basic Analysis}

We treat a dipole embedded in an infinite, homogeneous nonmagnetic $\left(\mu_{\text {rel }}=1\right)$ medium. The a-c quantities are described by their complex amplitudes. A column vector is expressed by a symbol $\mathbf{a}$ and its transpose, a row-vector, by ã. The Hermitian conjugate of a vector $\mathbf{a}$ is denoted by $a^{+}=\tilde{a}^{x}$. Symbols like aã, $\mathbf{k} \tilde{\mathbf{k}}$, etc. denote dyades; tensor quantities are denoted by symbols $\hat{a}, \hat{K}$, etc. In mks units Maxwell's equations take the form

$$
\begin{aligned}
& \nabla \times \mathbf{E}=-j \omega \mu_{0} \mathbf{H}-\mathbf{M} \\
& \nabla \times \mathbf{H}=j \omega \epsilon_{0} \hat{\epsilon} \mathbf{E}
\end{aligned}
$$

where the introduction of the magnetic current density $M$ will allow us to represent a small wire loop immersed in plasma by the equivalent magnetic dipole, with a distribution of magnetic current

$$
\mathbf{M}(\mathbf{r})=j \omega \mathbf{p}_{m} \delta(\mathbf{r})
$$

where $\delta(\mathbf{r})$ is Dirac's $\delta$-function.

In (1) we have dropped factors $e^{j \omega t}$ expressing the time dependence and $\hat{\epsilon}$ is of the form

$$
\hat{\epsilon}=\left\|\begin{array}{rrr}
\epsilon_{1} ; & -j \epsilon_{2} ; & 0 \\
j \epsilon_{2} ; & \epsilon_{1} ; & 0 \\
0 ; & 0 ; & \epsilon_{3}
\end{array}\right\|
$$


encountered in magneto-ionic theory. For the source-free $(\mathbf{M}=0)$ medium one easily finds, from Maxwell's equation, the wave equation which, when applied to waves of the form $\mathbf{E}=\mathbf{E}_{0} \exp$ $(-j \mathbf{k} \cdot \mathbf{r})$, leads to

$$
\hat{W}_{E}\left(\mathbf{k}, \mathbf{k}_{\mathbf{0}}\right) \mathbf{E}=0
$$

where

$$
\hat{W}_{E}\left(\mathbf{k}, \mathbf{k}_{0}\right)=\mathbf{k} \tilde{\mathbf{k}}-(\mathbf{k} \cdot \mathbf{k}) \hat{1}+k_{\hat{\sigma}}^{2} \hat{\epsilon}
$$

For nonvanishing fields one must have

$$
\operatorname{det} \hat{W}_{E}\left(\mathbf{k}, \mathbf{k}_{0}\right)=0 .
$$

One can easily show that, with $\epsilon$ of the form (3)

$$
\operatorname{det} \hat{W}_{E}=k_{0}^{2}\left(\epsilon, \sin ^{2} \theta+\epsilon_{3} \cos ^{2} \theta\right)\left(k^{2}-k_{I}^{2}\right)\left(k^{2}-\underset{I I}{2}\right)
$$

with refractive indices $k_{I}^{2} / k_{0}^{2}, k_{I I}^{2} / k_{0}^{2}$ for the ordinary and extraordinary wave given by

$$
\frac{k_{I, I I}^{2}}{k_{0}^{2}}=\frac{\left(\epsilon_{1}^{2}-\epsilon_{2}^{2}\right) \sin ^{2} \theta+\epsilon_{1} \epsilon_{3}\left(1+\cos ^{2} \theta\right) \pm\left[\left(\epsilon_{1}^{2}-\epsilon_{2}^{2}-\epsilon_{1} \epsilon_{3}\right)^{2} \sin ^{4} \theta+4 \epsilon_{2}^{2} \epsilon_{3}^{2} \cos ^{2} \theta\right]^{1 / 2}}{2\left(\epsilon_{1} \sin ^{2} \theta+\epsilon_{3} \cos ^{2} \theta\right)}
$$

\section{Dyadic Green's Functions and Their Fourier Transforms}

We want to express the radiation field in terms of a given source distribution. Since Maxwell's equations are linear we can certainly write

$$
\mathbf{E}(\mathbf{r})=\int d \mathbf{r}^{\prime} \hat{G}_{E M}\left(\mathbf{r}, \mathbf{r}^{\prime}\right) \mathbf{M}\left(\mathbf{r}^{\prime}\right) \quad \mathbf{H}(\mathbf{r})=\int d \mathbf{r}^{\prime} \hat{G}_{H M}\left(\mathbf{r}, \mathbf{r}^{\prime}\right) \mathbf{M}\left(\mathbf{r}^{\prime}\right)
$$

where $G_{E M}, G_{H M}$ are matrices which may suitably be called dyadic Green's functions. Once they are known, the problem is solved. We can obtain them by algebraic operations by means of Fourier analysis of the fields of the form

$$
\begin{gathered}
\mathbf{E}(\mathbf{r})=\int d \mathbf{k} \mathbf{E}_{\mathbf{k}} e^{-j(\mathbf{k} \cdot \mathbf{r})} \\
\mathbf{E}_{\mathbf{k}}=\frac{1}{8 \pi^{3}} \int d \mathbf{r E}(\mathbf{r}) e^{j(\mathbf{k} \cdot \mathbf{r})} .
\end{gathered}
$$

We also define matrices $\hat{g}_{E M}, \hat{g}_{H M}$, which are Fourier transforms of the dyadic Green's functions defined above:

$$
\hat{G}_{H M}=\frac{1}{8 \pi^{3}} \int d \mathbf{k} \hat{g}_{H M}(\mathbf{k}) e^{-j \mathbf{k}\left(\mathbf{r}-\mathbf{r}^{\prime}\right)}
$$

Introducing (10a, b) and (11) into (9) we obtain

$$
\begin{aligned}
& \mathbf{E}_{\mathbf{k}}=\hat{g}_{E M} \mathbf{M}_{\mathbf{k}} \\
& \mathbf{H}_{\mathbf{k}}=\hat{g}_{H M} \mathbf{M}_{\mathbf{k}} .
\end{aligned}
$$




\section{Computation of the Dyadic Green's Function Transforms}

We shall reduce the computation of the transforms $\hat{g}_{H M}, \hat{g}_{E M}$ to matrix operations. To do this, it is convenient to define a matrix

$$
\hat{K}=\left\|\begin{array}{ccc}
0, & -k_{3}, & k_{2} \\
k_{3}, & 0, & -k_{1} \\
-k_{2}, & k_{1}, & 0
\end{array}\right\|
$$

such that vector products may be written as matrix products, e.g.,

$$
\mathbf{k} \times \mathbf{E}=\hat{K} \mathbf{E} .
$$

With this notation the Fourier-transforms of Maxwell's equations may be written in the form

$$
\begin{gathered}
-j \hat{K} \mathbf{E}_{\mathbf{k}}=-j \omega \mu_{0} \mathbf{H}_{\mathbf{k}}-\mathbf{M}_{\mathbf{k}} \\
-j \hat{K} \mathbf{H}_{\mathbf{k}}=j \omega \epsilon_{0} \hat{\boldsymbol{\epsilon}} \mathbf{E}_{\mathbf{k}} .
\end{gathered}
$$

The determinant of the matrix $\hat{K}$ is clearly zero and therefore $\hat{K}$ does not possess an inverse. In most cases, however $\hat{\epsilon}^{-1}$ exists. We solve for $\mathbf{E}_{\mathbf{k}}$ and $\mathbf{H}_{\mathbf{k}}$ by multiplying equations (15) by $1 / j \omega \mu_{0}$ and $\hat{\epsilon} / j \omega \epsilon_{0}$ respectively and obtain

$$
\mathbf{H}_{\mathbf{k}}=\frac{1}{j \omega \mu_{0}}\left(j \hat{K} \mathbf{E}_{\mathbf{k}}-\mathbf{M}_{\mathbf{k}}\right) \quad \mathbf{E}_{\mathbf{k}}=\left(-\hat{\epsilon}^{-1} / j \omega \epsilon_{0}\right) j \hat{K} \mathbf{H}_{\mathbf{k}} .
$$

Substituting these results into (15) we obtain

$$
\left(\mathbf{k} \mathbf{k}-(\mathbf{k} \cdot \mathbf{k} \cdot) \hat{1}+k_{0}^{2} \hat{\epsilon}\right) \mathbf{E}_{\mathbf{k}}=-j \hat{K} \mathbf{M}_{\mathbf{k}} \quad\left(\hat{K} \hat{\epsilon}^{-1} K+k_{0}^{2}\right) \mathbf{H}_{\mathbf{k}}=j \omega \epsilon_{0} \mathbf{M}_{\mathbf{k}},
$$

since

$$
\hat{K} \hat{K}=\mathbf{k} \mathbf{k}-(\mathbf{k} \cdot \mathbf{k}) \hat{1}
$$

Comparing (17) with (12) we find the dyadic Green's function transforms

$$
\hat{g}_{E M}=-j \hat{W}_{E}^{-1} \hat{K}, \quad \hat{g}_{H M}=j \omega \epsilon_{0} W_{H}^{-1}
$$

where $\hat{W}_{E}$ is defined by (5) and $\hat{W}_{H}$ is given by

$$
\hat{W}_{H}=\hat{K} \hat{\epsilon}^{-1} \hat{K}+k_{0}^{2}
$$

We shall, in the following, only need $\hat{g}_{H M}$. For this reason, and also since the expressions for $\hat{W}_{E}^{-1}$ have already been given in Kogelnik [1960a], we give below only $\hat{W}_{H}^{-1}$. One finds, putting $\mathbf{k}=k \mathbf{n}$ where $\mathbf{n}$ is a unit vector

$$
\hat{W}_{H}^{-1}=\frac{\tilde{\mathbf{n}} \hat{\boldsymbol{\epsilon}} k^{4} \mathbf{n} \tilde{\mathbf{n}}-k^{2} k_{0}^{2} \hat{S}+k_{0}^{4} \hat{1}(\operatorname{det} \hat{\boldsymbol{\epsilon}})}{\operatorname{det} \hat{W}_{E}}
$$


where

$$
\begin{array}{ll}
S_{11}=\epsilon_{1} \epsilon_{3}+n_{1}^{2}\left(\epsilon_{1}^{2}-\epsilon_{2}^{2}\right) & S_{21}=j n_{3}^{2} \epsilon_{2} \epsilon_{3}+n_{1} n_{2}\left(\epsilon_{1}^{2}-\epsilon_{2}^{2}\right) \\
S_{12}=-j n_{3}^{2} \epsilon_{2} \epsilon_{3}+n_{1} n_{2}\left(\epsilon_{1}^{2}-\epsilon_{2}^{2}\right) & S_{22}=\epsilon_{1} \epsilon_{3}+n_{2}^{2}\left(\epsilon_{1}^{2}-\epsilon_{2}^{2}\right) \\
S_{13}=j n_{2} n_{3} \epsilon_{2} \epsilon_{3}+n_{1} n_{2} \epsilon_{1} \epsilon_{3} & S_{23}=n_{2} n_{3} \epsilon_{1} \epsilon_{3}-j n_{1} n_{3} \epsilon_{2} \epsilon_{3} \\
S_{31}=-j n_{2} n_{3} \epsilon_{2} \epsilon_{3}+n_{1} n_{3} \epsilon_{1} \epsilon_{3} & \\
S_{32}=n_{2} n_{3} \epsilon_{1} \epsilon_{3}+j n_{1} n_{3} \epsilon_{2} \epsilon_{3} & \\
S_{33}=2 n_{3}^{2} \epsilon_{1} \epsilon_{3}+\left(n_{1}^{2}+n_{2}^{2}\right)\left(\epsilon_{1}^{2}-\epsilon_{2}^{2}\right) .
\end{array}
$$

\section{Power Radiated by a Distribution of Magnetic Current}

The power radiated by a volume element $d x d y d z$ of the distribution is given by Poynting's

$$
d P=-\frac{1}{2} \mathbf{H}^{x} \mathbf{M} d x d y d z
$$

Hence the power radiated by the magnetic current system is given by the integral

$$
P=-\frac{1}{2} \int d \mathbf{r}^{\prime} \int d \mathbf{r} \mathbf{M}^{+}\left(\mathbf{r}^{\prime}\right) \hat{G}_{H M}^{+} \mathbf{M}(\mathbf{r})
$$

over the magnetic current distribution which, in terms of the dyadic Green's function, transforms can be shown to reduce to

$$
P=-4 \pi^{3} \int d \mathbf{k}\left(\mathbf{M}_{\mathbf{k}}^{+} \hat{g}_{H M} \hat{M}_{\mathbf{k}}\right)^{x}
$$

by the application of well-known $\delta$-function identities.

\section{Radiation Admittance of a Current Loop}

As mentioned in the first section, the magnetic dipole is represented by a magnetic current density distribution

$$
\mathbf{M}(\mathbf{r})=j \omega \mathbf{p}_{m} \delta(\mathbf{r}) .
$$

This corresponds to an oscillating current in a small loop such that the dipole moment $p_{m}$ is given by $p_{m}=\mu_{0} I a^{2} \pi, I=I_{0} \sin \omega t$ where $a$ is the radius of the loop. This has an effect different from that produced by a rotating electron in its orbit, which is equivalent to two linearly polarized oscillating currents with a phase difference of $\pi / 2$. We have shown in Kogelnik [1960b] that the power radiated by two such linearly polarized currents is, in a gyroelectric medium, not equivalent to the sum of the power of the separately excited individual antennas (see appendix).

The spatial Fourier-transform of the magnetic current density is given by

$$
\mathbf{M}_{\mathbf{k}}=\frac{j \omega \mathbf{p}_{m}}{8 \pi^{3}}
$$

By application of (23) we find that the complex conjugate of the power can be written as a bilinear form

$$
P^{x}=\frac{\omega^{2}}{2} \mathbf{p}_{m}^{+} \hat{z}_{m} \mathbf{p}_{m}
$$


with

$$
\hat{z}_{m}=-\frac{1}{8 \pi^{3}} \int d \mathbf{k} \hat{g}_{H M}
$$

The elements of $\hat{z}_{m}$ are measured in $\mathrm{ohm}^{-1} \mathrm{~m}^{-2}$ (admittance-units per unit area.) The matrix $z_{m}$ is split into its Hermitian and its anti-Hermitian part

$$
\hat{z}_{m}=\hat{r}_{m}+j \hat{x}_{m}
$$

and the real power radiated is

$$
P_{r}=\frac{\omega^{2}}{2} \mathbf{p}_{m}^{+} \hat{r}_{m} \mathbf{p}_{m}
$$

We can substitute in (26) for $g_{H M}$ from (18) with the result

$$
\hat{z}_{m}=\frac{-j \omega \epsilon_{0}}{8 \pi^{3} k_{0}^{2}} \int_{0}^{\pi / 2} \sin \theta d \theta \int_{-\infty}^{\infty} d \mathbf{k} \int_{0}^{2 \pi} d \phi \frac{k^{2}\left[\tilde{\mathbf{n}} \hat{\epsilon} \mathbf{n} k^{4} \mathbf{n} \tilde{\mathbf{n}}-k^{2} k_{0}^{2} \hat{S}+k_{0}^{4} \hat{1}(\operatorname{det} \hat{\boldsymbol{\epsilon}})\right]}{\tilde{\mathbf{n}} \hat{\epsilon} \mathbf{n}\left(k^{2}-k_{\mathrm{I}}^{2}\right)\left(k^{2}-k_{\mathrm{II}}^{2}\right)} .
$$

So far the theory is perfectly general and would also apply to lossy media. We now specialize the theory to apply to a lossless medium by assuming that $\epsilon_{1}, \epsilon_{2}$, and $\epsilon_{3}$ are real. Our numerical results apply to this case only. The matrices $\hat{M}_{m}, \hat{N}$ are defined by

$$
\begin{aligned}
& \hat{N}=\frac{1}{\pi} \int_{0}^{2 \pi} \mathbf{n} \tilde{\mathbf{n}} d \phi=\left\|\begin{array}{rrr}
\sin ^{2} \theta ; & 0 ; & 0 \\
0 ; & \sin ^{2} \theta ; & 0 \\
0 ; & 0 ; & 2 \cos ^{2} \theta
\end{array}\right\| \\
& \hat{M}_{m}=\hat{M}_{m}^{+}=\frac{1}{\pi} \int_{0}^{2 \pi} S d \phi \\
& =\left\|\begin{array}{lrr}
2 \epsilon_{1} \epsilon_{3}+\left(\epsilon_{1}^{2}-\epsilon_{2}^{2}\right) \sin ^{2} \theta ;-2 j \epsilon_{2} \epsilon_{3} \cos ^{2} \theta & ; & 0 \\
2 j \epsilon_{2} \epsilon_{3} \cos ^{2} \theta & ; \quad 2 \epsilon_{1} \epsilon_{3}+\left(\epsilon_{1}^{2}-\epsilon_{2}^{2}\right) \sin ^{2} \theta ; & 0 \\
0 ; & 0 ; & 4 \epsilon_{1} \epsilon_{3} \cos ^{2} \theta+2\left(\epsilon_{1}^{2}-\epsilon_{2}^{2}\right) \sin ^{2} \theta
\end{array}\right\| .
\end{aligned}
$$

Having performed the integrations with respect to $\phi$ and $k$, the final results can be written in the form

$$
r_{m}=-\frac{\pi}{2 Z_{0} \lambda_{0}^{2}} \int_{0}^{1} \frac{d u F_{m}}{k_{0}^{3}\left[\epsilon_{1}+\left(\epsilon_{3}-\epsilon_{1}\right) u^{2}\right]\left(k_{\mathrm{I}}^{2}-k_{\mathrm{II}}^{2}\right)}
$$

with $Z_{0}=\left(\mu_{0} / \epsilon_{0}\right)^{1 / 2}, \lambda_{0}=\frac{2 \pi}{k_{0}}$ and $\hat{F}_{m}$ being the Hermitian part

$$
F_{m}=\operatorname{Herm}\left\{\tilde{\mathbf{n}} \hat{\boldsymbol{n}}\left(k_{\mathrm{I}}^{5}-k_{\mathrm{II}}^{5}\right) \hat{N}-k_{0}^{2}\left(k_{\mathrm{I}}^{3}-k_{\mathrm{II}}^{3}\right) \hat{M}_{m}+2 k_{0}^{4}\left(k_{\mathrm{I}}-k_{\mathrm{II}}\right) \hat{1}(\operatorname{det} \hat{\boldsymbol{\epsilon}})\right\} .
$$

Thus the matrix $\hat{r}_{m}$ has the form

$$
\hat{r}_{m}=\left\|\begin{array}{lll}
\hat{r}_{m 1}, & -j r_{m 2}, & 0 \\
j r_{m 2}, & r_{m 1}, & 0 \\
0, & 0, & r_{m 3}
\end{array}\right\|
$$


with the elements

$$
r_{m i}=-\frac{\pi}{2 Z_{0} \lambda_{0}^{2}} \int_{0}^{1} \frac{d u f_{m i}(u)}{k_{0}^{3}\left[\epsilon_{1}+\left(\epsilon_{3}-\epsilon_{1}\right) u^{2}\right]\left(k_{\mathrm{I}}^{2}-k_{\mathrm{II}}^{2}\right)}
$$

where $u=\cos \theta$

$f_{m 1}=\operatorname{Re}\left\{\tilde{\mathbf{n}} \hat{\epsilon} \mathbf{n}\left(k_{\mathrm{I}}^{5}-k_{\mathrm{II}}^{5}\right) \sin ^{2} \theta-k_{0}^{2}\left(k_{\mathrm{I}}^{3}-k_{\mathrm{II}}^{3}\right)\left[2 \epsilon_{1} \epsilon_{3}+\left(\epsilon_{1}^{2}-\epsilon_{2}^{2}\right) \sin ^{2} \theta\right]+2 k_{0}^{4}\left(k_{\mathrm{I}}-k_{\mathrm{II}}\right) \operatorname{det} \hat{\epsilon}\right\}$

$f_{m 2}=\operatorname{Re}\left\{-2 k_{0}^{2}\left(k_{\mathrm{I}}^{3}-k_{\mathrm{II}}^{3}\right) \epsilon_{2} \epsilon_{3} \cos ^{2} \theta\right\}$

$f_{m 3}=\operatorname{Re}\left\{2 \tilde{\mathbf{n}} \hat{\epsilon} \mathbf{n}\left(k_{\mathrm{I}}^{5}-k_{\mathrm{II}}^{5}\right) \cos ^{2} \theta-2 k_{0}^{2}\left(k_{\mathrm{I}}^{3}-k_{\mathrm{II}}^{3}\right)\left[2 \epsilon_{1} \epsilon_{3} \cos ^{2} \theta+\left(\epsilon_{1}^{2}-\epsilon_{2}^{2}\right) \sin ^{2} \theta\right]+2 k_{0}^{4}\left(k_{\mathrm{I}}-k_{\mathrm{II}}\right) \operatorname{det} \hat{\epsilon}\right\}$.

\section{Numerical Data Concerning the Elements of the Admittance Matrix}

Since the expressions (35) for the admittance matrix are rather cumbersome, we have evaluated them by means of a digital computer (Ferranti Mercury). Table 1 gives the element $r_{m 1}$. Table 2 records values for $r_{m 2}$, while $r_{m 3}$ is given in table 3 . The entries in these tables must be

TABLE 1

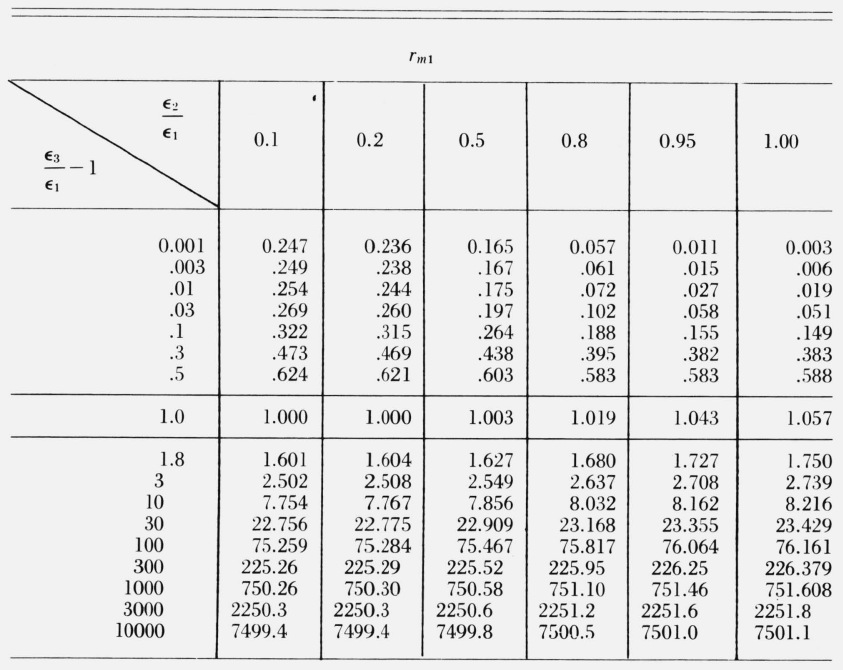

TABLE 2

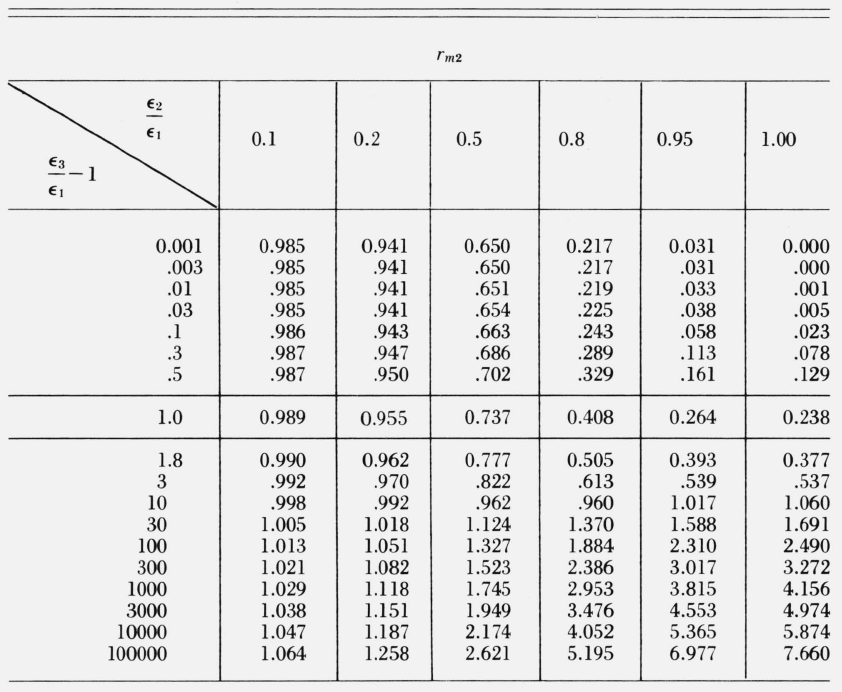


TABLE 3

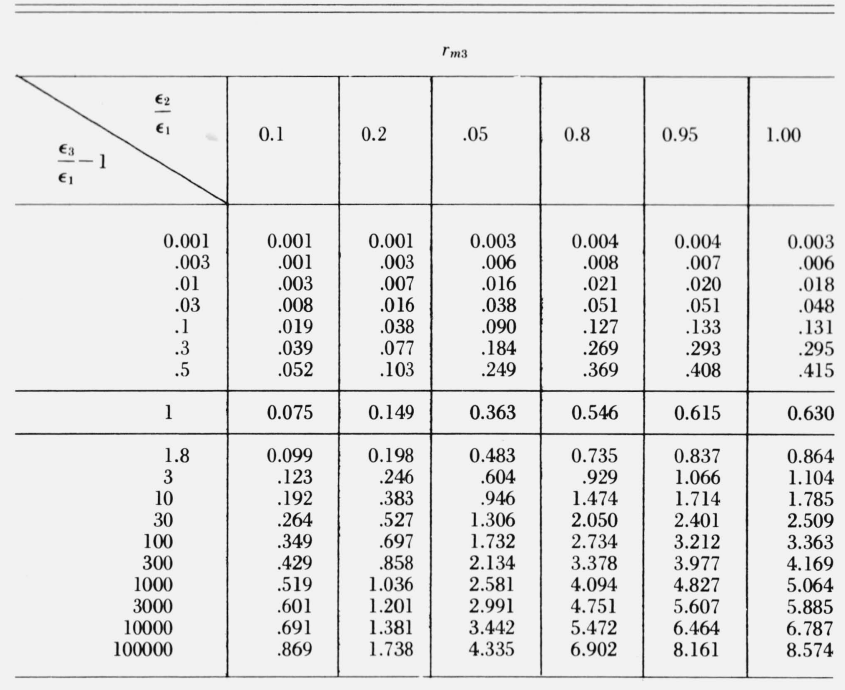

multiplied by

$$
\frac{2 \pi}{3 Z_{0} \lambda_{0}^{2}} \epsilon_{1}^{3 / 2} \mathrm{mhos} / \mathrm{m}^{2}
$$

where $Z_{0} \underline{\Omega} 120 \pi \mathrm{ohm}$, and $\lambda_{0}$ is the free space wavelength. The values so obtained are expressed in $\mathrm{mhos} / \mathrm{m}^{-2}$. We also give graphs of these quantities (figs. 1, 2, 3, 4, 5, 6).

The power can be computed from the formula

$$
P_{r}=\frac{\omega^{2}}{2} \mathbf{p}_{m}^{+} \hat{r}_{m} \mathbf{p}_{m}
$$

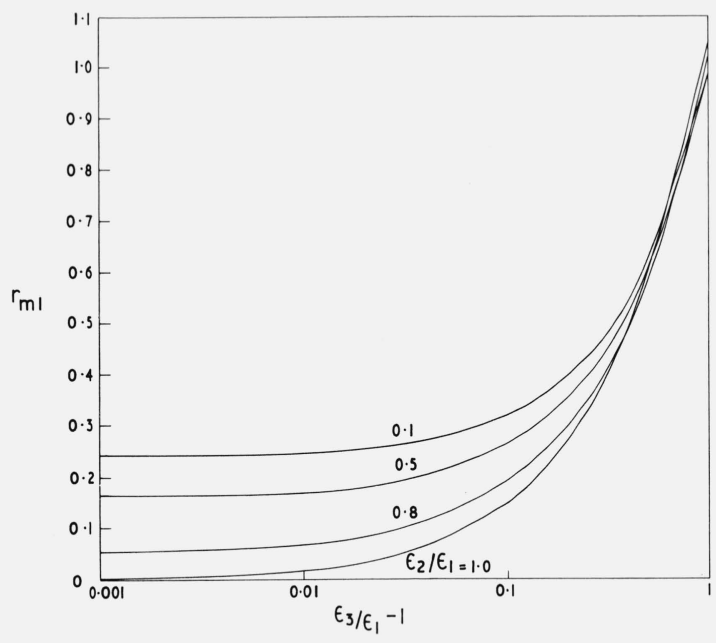
FIGURE 1. Matrix element $\mathrm{r}_{\mathrm{m} 1}$ as a function of $\frac{\epsilon_{3}}{\epsilon_{1}}-1$
with $\epsilon_{2} / \epsilon_{1}$ as a parameter.

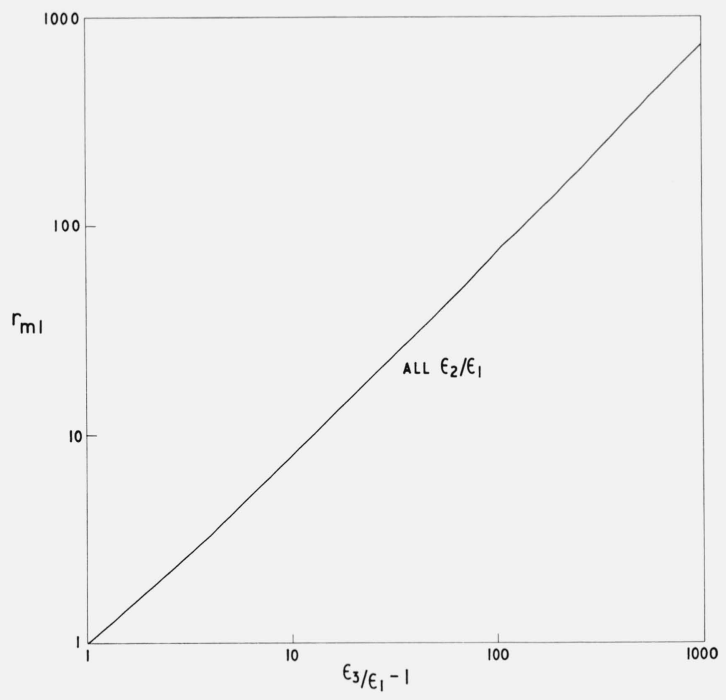

FIGURE 2. Matrix element $\mathrm{r}_{\mathrm{m} 1}$ as a function of $\frac{\epsilon_{3}}{\epsilon_{1}}-1$ in the range where it does not depend on the ratio $\epsilon_{2} / \epsilon_{1}$. 


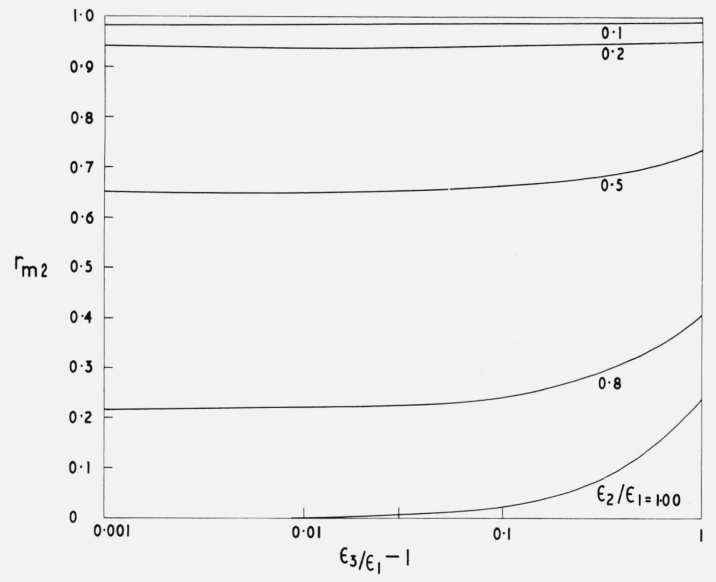

Figure 3. Matrix element $\mathrm{r}_{\mathrm{m} 2}$ of the admittance matrix as a function of $\frac{\epsilon_{3}}{\epsilon_{1}}-1$ with $\epsilon_{2} / \epsilon_{1}$ as a parameter.

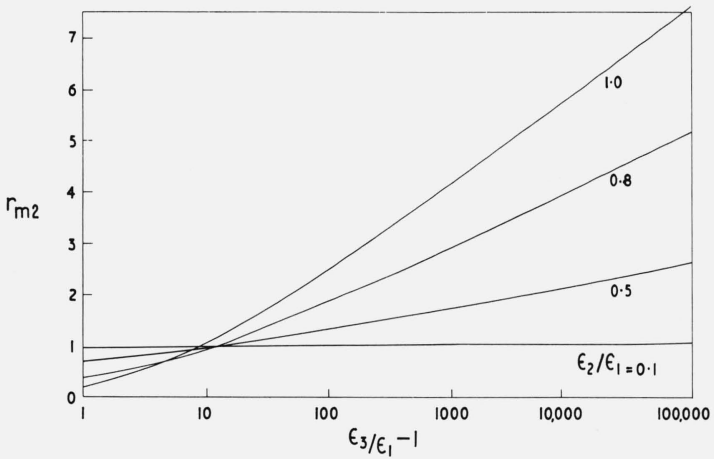

FIGURE 4. Matrix element $\mathrm{r}_{\mathrm{m} 2}$ as a function of $\frac{\epsilon_{3}}{\epsilon_{1}}-1$ with $\epsilon_{2} / \epsilon_{1}$ as a parameter.

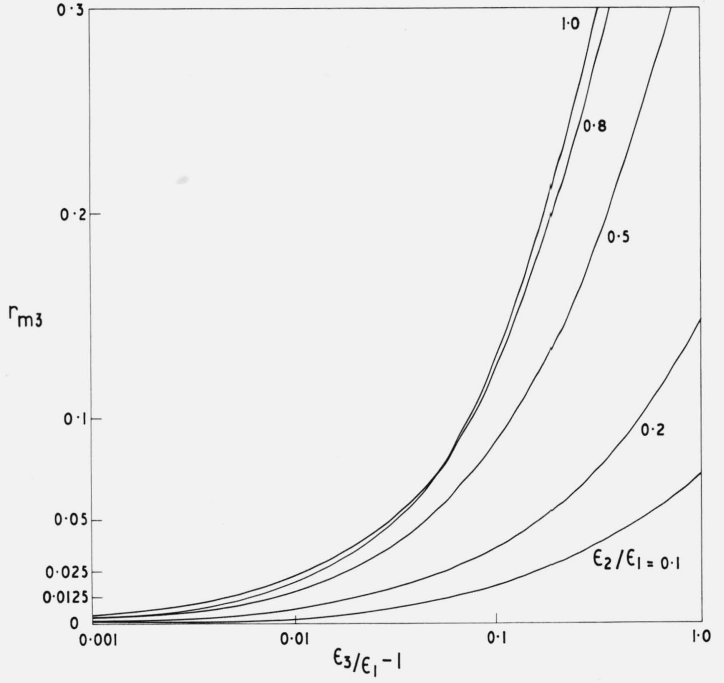

Figure 5. Matrix element $\mathrm{r}_{\mathrm{m} 3}$ of the admittance as a function of $\frac{\epsilon_{3}}{\epsilon_{1}}-1$ with $\epsilon_{2} / \epsilon_{1}$ as a parameter.

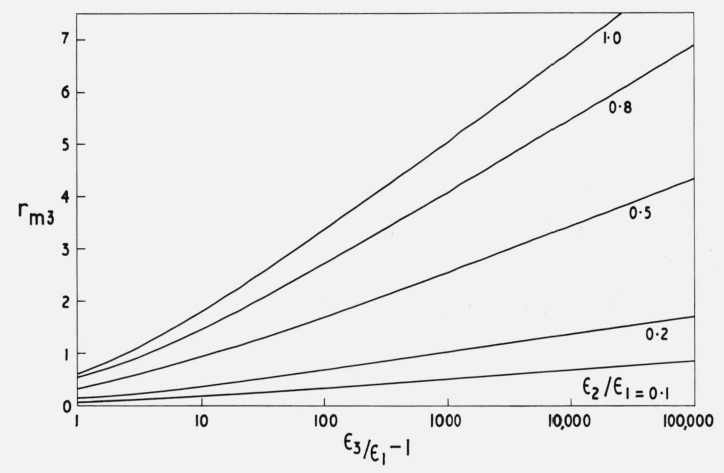

Figure 6. Matrix element of the admittance as a function of $\frac{\epsilon_{3}}{\epsilon_{1}}-1$ with $\epsilon_{2} / \epsilon_{1}$ as a parameter.

The $z$-axis (3-axis) was chosen throughout as the direction of the applied magnetic field so that in the special case of a dipole oriented parallel to the magnetic field, $\mathbf{p}$ is given by

$$
\mathbf{p}=\left(\begin{array}{r}
0 \\
0 \\
p_{z}
\end{array}\right) p^{+}=\left(0,0, p_{z}\right)
$$

and the expression for the power reduces to

$$
P_{r}=\frac{\omega^{2}}{2} r_{3}\left(p_{z}\right)^{2}
$$

\section{Conclusion}

The data of this paper should be used with caution. When the refractive index of the cold plasma theory tends to infinity, the wavelength tends to zero, thus invalidating the assumption that the current loop is small. The reactive power, in any case, diverges. It has no meaning 
for an elementary dipole or for any $\delta$-function distribution of current over a conducting surface.

When there is no propagation, the radiation admittance is zero.

The author thanks Mrs. R. Baumberg for carrying out the computing and Mr. E. Gilbert for checking results.

The research reported in this document has been sponsored in part by the United States Air Force, under Grant 63-33 and monitored through the European Office, ARDC.

\section{Appendix 1}

It was shown in Kogelnik [1960a, 1960b] that the real power radiated by an electric dipole with moment $p$ is given by an expression

$$
P=\frac{\omega^{2}}{2} \overrightarrow{\mathbf{p}}^{+}\left\|\begin{array}{llr}
r_{x} & -j r_{y} & 0 \\
j r_{y} & r_{x} & 0 \\
0 & 0 & r_{z}
\end{array}\right\| \overrightarrow{\mathbf{p} .}
$$

Thus for the special case of dipoles which are linearly polarized in directions parallel to $x$ or to $y$ the power is given by

$$
P_{x}=\frac{\omega^{2}}{2} r_{x}\left|p_{x}\right|^{2} \quad \text { or } P_{y}=\frac{\omega^{2}}{2} r_{y}\left|p_{y}\right|^{2} \text { respectively. }
$$

On the other hand, for the case of a circularly polarized moment with

$$
p_{z}=0, \pm j p_{x}=p_{y}
$$

we obtain

$$
P_{c}=\omega^{2}\left|p_{x}\right|^{2}\left(r_{x} \pm r_{y}\right)
$$

and this is not the sum of the powers radiated by two aerials when each is excited in the absence of the other. The radiation of the aerials shows 'interference' caused by the coupling due to the medium.

\section{References}

Kogelnik, H. (1960a), On electromagnetic radiation in magneto-ionic media, J. Res. NBS 64D (Radio Prop.), No. 5, 515-523. Kogelnik, H. (Aug. 1960b), The radiation resistance of an elementary dipole in anisotropic plasmas, Fourth Intern. Conf. on Ionization Phenomena in Gases, Uppsala, Sweden (North-Holland Publishing Co., Amsterdam).

Kogelnik, H., and H. Motz (1963), Electromagnetic radiation from sources embedded in an infinite anisotropic medium and the significance of the Poynting vector, Proc. Symposium on Electromagnetic Theory and Antenna, Copenhagen, 1962, pp. 477-493, ed. E. C. Jordan (Pergamon Press, Oxford, Eng.).

(Paper 69D5-502) 